\title{
PLANNING AND DESIGNING WEB-BASED ELECTRONIC COMMERCE: A CASE STUDY IN THE INSURANCE INDUSTRY
}

\author{
Rolf Alexander Teubner, Stefan Klein \\ Institute for Information Systems, University of Muenster, Muenster, Germany \\ wialte@wi.uni-muenster.de
}

\begin{abstract}
Electronic Commerce (EC) comprises new ways of doing business with customers, suppliers and other business partners. EC is enabled by information and communication technology, in particular the World Wide We (in short, the Web). While numerous organisations have started to use the Web as an interface to clients and business partners, many have been disappointed by lower than expected response rates and higher than expected costs. One reason for non profitable Web based EC applications is that they do not reflect the strategic preconditions of EC. This lack may be due to deficits in the process of developing an EC-strategy for their Web application. Furthermore, there is a void of instruments and tools to support this process. In order to fill this void, this paper proposes two basic frameworks for planning a Web based EC application. The application of these frameworks is illustrated by the example of a medium seized insurance company. Based on experiences drawn from the case-study, the proposed frameworks will be evaluated.
\end{abstract}

\section{INTRODUCTION}

Scarcely a platform has been tackled as intensively as the World Wide Web in discussions about the value of new media. Euphoric statements have motivated companies to explore this medium for the purpose Electronic Commerce. Because getting onto the Web is not expensive, many of these companies have decided to offer a simple presentation but results are often not satisfactory. One reason for non profitable Web based EC applications (shorter: Web applications) is that they do not reflect a clear strategic rationale and that their position in relation to other media and functional areas is not clear. On the one hand the potential of the Web as an instrument for marketing and sales is not exploited, on the other hand organisational and technical conditions of the enterprise are not taken into account. It is the intention of this paper to point out the most relevant aspects that have to be considered and provide a guideline for the process of planning a Web site and designing the corresponding Web application.

\section{FUNDAMENTAL FRAMEWORKS FOR PLANNING WEB-BASED EC}

On an operational level four basic parameters have to be considered when designing a Web application. These parameters are the content, features, layout and the technologies used to implement the application.

Content: The content of a Web application comprises the information presented to the visitor of a Web site. The Web offers hypermedia representation of information with few restrictions in the amount of content that is provided. These qualities are reflected in markedly different profiles for Web advertising in contrast to traditional advertisements, which would gain little or no attention on the Web.

Features: Web applications in most cases offer more than store front representation of information. Often applications offer opportunities to communicate by e-mail, to send information in predefined forms; to do some database query or transaction processing. All these functions besides information representation are covered by the parameter "features".

Layout: The layout defines the interface which gives the user access to information content and functionality. Layout design determines how user-friendly an application is and therefore has an impact on the level of user acceptance. The Web as a new and emerging medium provides a plethora of design opportunities that are only gradually understood. Experts in previous media, such as print, often show difficulties to capture the richness and specific features of this new medium and to develop visually attractive and operationally efficient solutions.

Technology: While the design parameters above define the application conceptually, all (software-) technical choices are comprised by "technology". First, the software architecture has to be defined. A typical architecture consists of a database server, a Web server and dedicated software for electronic commerce. Dedicated EC functions such as electronic catalogues, shopping baskets, customer profile management, payment and statistics are integrated in software packages called commerce or merchant servers. Based on the architectural decisions, the technologies for implementation can be chosen. Typical technologies for Web applications are HTML and VRML for information presentation, Java, Java Script, and CGI for computing and database access, and dedicated protocols for application and secure transaction services. 
Although there are some general guidelines for Web application design, not only the conceptual design of the application determines its success. Rather the way the application is strategically positioned, organisationally anchored and technically integrated has an even stronger impact on success. These factors can be seen as context variables that have to be taken into account when planning the application. The model in figure 1 tries to point out the relationship between these context factors influencing Web design and the design parameters (adapted from Klein 1997).

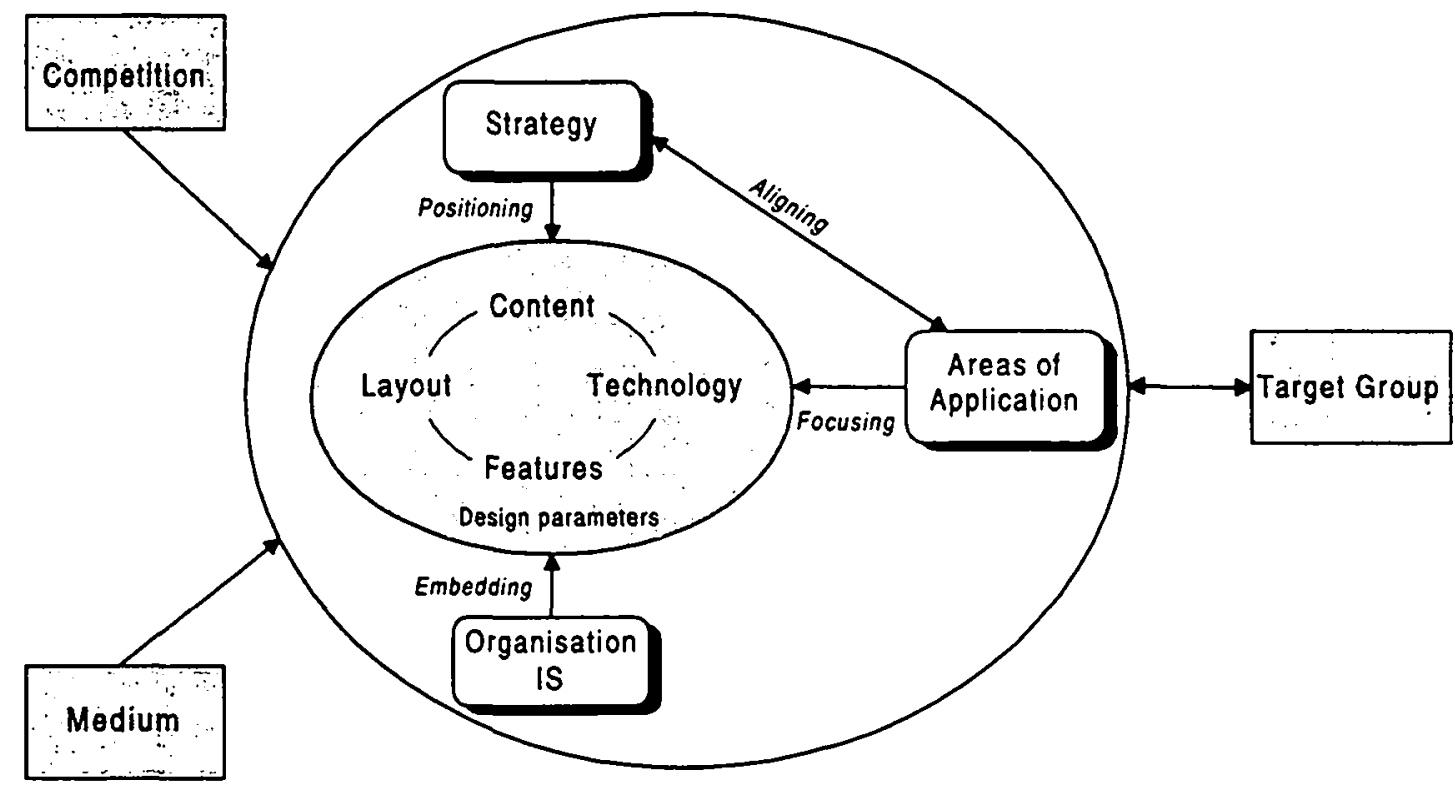

Figure 1: Factors influencing Web design

The model in figure 1 can be seen as a contingency model. It suggests interdependencies between context and design that have to be considered when planning a Web strategy, but it does not imply deterministic relationships between context variables and the design parameters. The latter are enclosed in the inner ellipse in figure 1 while the former are outside this grey area. The context variables can be distinguished in external context and internal context variables. The external variables are not inside the borders of the enterprise (indicated by the large circle), while the internal variables are. The external variables are competition, target group and medium.

Competition does not only cover competing enterprises but also their Web approaches. Because Web applications are highly visible and can be compared easily, the competition of imitation has intensified. It is a main challenge for an enterprise to find a Web approach that differs from those of other companies, supports the strategic profile of the enterprise and facilitates a sustainable advantage.

Target group: A fundamental distinction among target groups is reflected in the terms business-to-consumer electronic commerce $(b 2 c-E C)$ and business-to-business electronic commerce (b2b-EC). While the first term refers to the processes carried out between enterprise and consumer, the second one refers to transactions with suppliers and other institutional business partners. It is obvious that conditions and requirements are different in both cases. For example, b2b-relationsships are in general less anonymous and more stable than b2crelationships, integration with client information systems is possible etc. Beyond the crucial decision whether existing or new business partners or customers shall be addressed, the known segmentation approaches from marketing can be applied accordingly.

Medium: In general, each Web application is restricted or enabled by the characteristics of the medium "Web". The Web technology determines what is technically feasible. New technologies such as Java applets, script languages and dynamic generation of HTML have expanded the possible range of features of Web applications. The new transport protocol version, TCP/IPv6, is going to resolve some of the shortcomings of the current protocol, namely facilitating bandwidth guarantees.

In contrast to the external context, the inner context variables are in the decision scope of the enterprise. The strategy, the organisational structure, the information systems and the area of the application are results of management decisions. But as these decisions are made before Web application development, they are preconditions and have to be taken into consideration when developing a Web strategy. 
Strategy: The Web strategy is part of the information strategy of the enterprise, which itself is closely linked to its business strategy. The information strategy is a complex set of explicit visions, goals and guidelines with respect to the demand and supply of information necessary to conduct the business. The Web strategy is that part of the information strategy which refers to the usage of the Web in delivering and accessing information in order to leverage business opportunities (Lederer et al. 1997).

Organisational structure / information systems: As far as a Web application exceeds store front presentation of information and offers dynamical information content, communication or transaction functionality, there is a great need to integrate it organisationally and technically. From an organisational point of view, the application has to be integrated into business processes, in case of a customer interface, e.g., order fulfilment or invoice management. Furthermore, rules for maintenance and updating have to be established. From a technical point of view, interfaces for access to internal databases, to communication systems or to workflow management systems have to be defined.

Areas of application: The areas of application define the purpose and scope of the application. Examples are advertising, providing value added services, delivery of (digital) products or simplifying administration tasks.

Though the framework in figure 1 is descriptive in its nature, it summarises and structures success factors for a Web application. Those success factors can be transformed into a development cycle model of Web-applications as depicted in figure 2 (see Klein 1998).

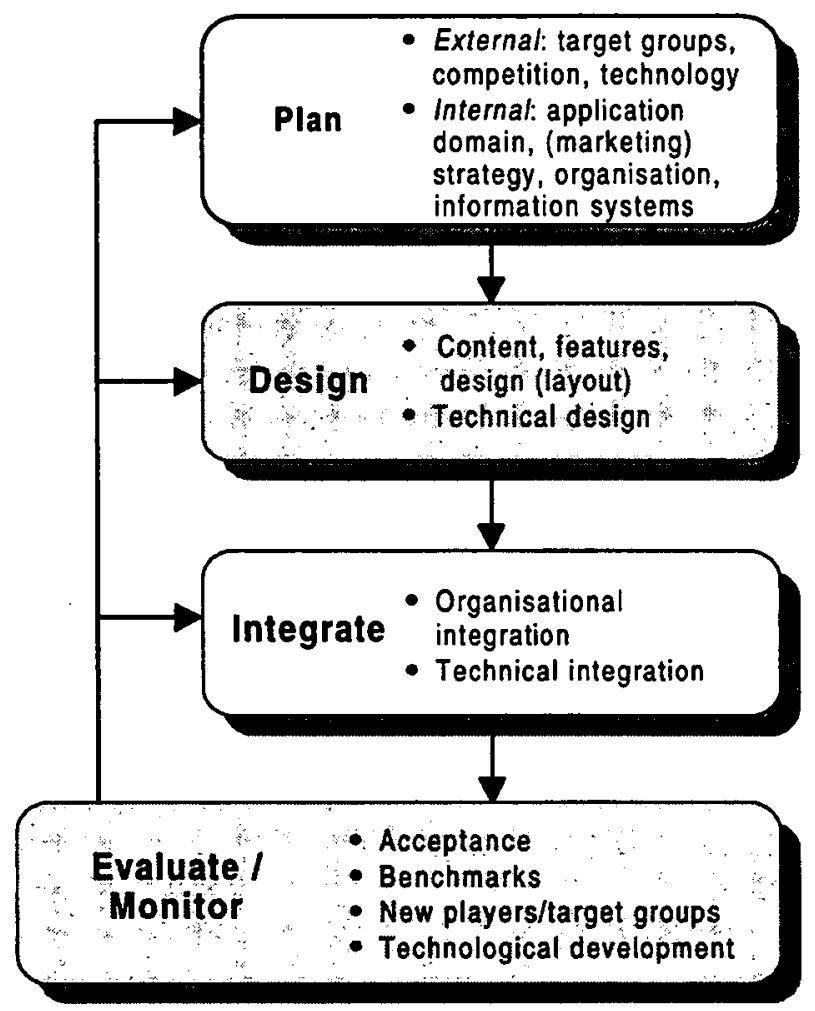

Figure 2: Web Engineering Development Cycle Model

This paper gives a detailed description of a concrete application of the proposed development cycle model (figure 2) and the underlying framework (figure 1). For this reason, the model will only be outlined at this point:

- The planning phase covers the analysis of the external situation, the strategic positioning and the marking out of application areas. The outcome of the planning phase is a concrete definition of what shall be achieved with the Web site. This definition can be expressed in terms of a Web strategy, that commits the objectives of the application as well as its scope.

- The design phase covers all decisions that aim at defining how the aims planned shall be accomplished. These decisions refer to the design parameters content, layout, features and technologies. The result of the design phase is a conceptual and technical specification of the Web application.

- The implementation of the application is done in the integration phase. But the challenge of this phase is less to derive code from the design specification but to embed the application into the enterprise infrastructure. Besides coding, the technical and organisational interfaces to the internal infrastructure have to be built. In numerous cases, the adaptation of the infrastructures themselves will be necessary, too. 
- Due to the highly dynamical development of the Web and its usage, Web applications require continuous improvement efforts as soon as they are established. This is done during the evaluation phase. Observing competitors' activities, customer reactions and technology development aims at identifying the need for actualisation, adaptation, or enlargement of the Web site. The regular analysis of competitors' Web sites usually gives a good, albeit not sufficient, insight into competitors actions.

\section{DEVELOPING A WEB-APPLICATION: THE CASE OF AN INSURANCE COMPANY}

The development project that is described here was conducted in co-operation between a project group , consisting of seven students and one research assistant from the University of Münster, Institute of Information Systems, and an insurance company called Landwirtschaftlicher Versicherungsverein Münster (LVM). The LVM is a medium-seized company, well established in the German insurance market. It employs about 2000 employees, and has about 2.8 Million customers. The annual revenue is DEM 3.4 billion.

The project started in the end of 1997. At this time, LVM had already a Web presence. The design and concept, however, had mainly been done by an external advertising company that was also in charge of the maintenance of the application. These first Web activities were mainly motivated by the interest for the new medium "World Wide Web" and the concern not to be left behind by technology leaders. This first initiative reflected more a "me-too"-approach than a convincing strategic rationale. As a consequence, success fell far from expectations.

Besides the problem, that LVM did not pursue a convincing Web-strategy, there were problems in making full use of the Web. The service provider for the LVM was experienced in the use of traditional marketing media but had little experience with the Web and were not fully aware of the profound differences between traditional media and the Web (Kassaye 1997). The most striking difference is, that the Web is an interactive medium in contrast to print media, broadcasting or television that enable only one-way distribution of information. Other differences concern the integration of the Web strategy into the overall marketing and communication strategy. While, for example, a corporate design is a necessity from a marketing communications point of view, it has been discussed controversially, whether the Web presence, which often pursues a slightly different strategy than the traditional media, should follow the same principles and regulations.

As a response to these problems, the LVM decided to develop a new, more convincing Web strategy. Furthermore, LVM put the provision of the Web services into the hands of another provider. The new Web strategy was developed in co-operation with the University of Muenster. The common project was conducted according to the process model shown in figure 2 .

\section{Planning phase}

During the planning phase, the external context, i. e. the industry, was examined. Business strategies, Web usage in the industry and Web strategies of relevant competitors were taken into account, when positioning the LVM's Web approach. Then, the areas of application were defined with respect to LVM's business, customers and infrastructures.

\section{External context analysis \\ Customers and Competitors: Industry situation and trends}

The insurance industry is just going through a process of fundamental change. A first trend is market saturation: While in the fifties market growth was nearly $15 \%$ a year, it was only just $3 \%$ in 1996 (Hackenberg; Reiter 1997). With diminishing demand-pull, the traditional markets have become more and more customer dominated. In the past, strong relationships bound customers to their insurance companies. But customers have become more sensitive to price and quality. Many of them do not mind to change their insurer for better products or conditions any longer.

A second trend is fierce competition. This trend is partly stimulated from market saturation and customer power. The insurers natural reaction to sinking profits in a shrinking market is increased competition within the industry. Another important factors stimulating competition is, that suppliers from other lines of business enter the insurance market. First, there are banks and companies offering financial services. These new players intend to offer customers full service packages including insurance and financial services, a strategy called "Allfinanz". Furthermore there is new competition by large independent insurance agents that reverse integrate their business. Other new competitors are, for example, car manufacturers that offer car insurance services together with their products.

Globalisation, the third major trend, is in a pull and push relation to competition. Globalisation is mainly enabled by the deregulation of the local insurance markets and the establishment of a common European market with a common currency, the Euro. Through globalisation, some great foreign insurance companies try to establish business on the German market, which is an attractive market with a total volume of DEM 250 billions 
(Hackenberg, Reiter 1997). On the other hand, globalisation gives large German companies access to foreign markets. A great potential is seen especially in emerging markets in Eastern Europe, Asia and Latin America. While international markets are only a potential for the large companies, there are also some opportunities for smaller companies that only have access to the national market. New potential is expected from new risks that need to be insured such as caused by sinking trust in pension insurance or risks of software. These new opportunities will be exploited by those companies, that quickly and flexibly respond to the emerging needs and offer innovative products fitting them.

The necessary reactions to the trends stated above that can be seen as success factors in the actual situation of the insurance industry, are:

- a shift from product orientation to customer orientation,

- market segmentation (esp. household vs. industry),

- differentiation of distribution channels according to market segments,

- development of new, individualised products,

- bundling and tailoring products,

- more emphasis on financial services and financial market functions,

- drawing to foreign markets,

- cost reduction.

The Web can help address most of these success factors. Information can be tailored to customers needs, if necessary even a segment-of-one-marketing is possible. The Web is a new distribution channel. Insurance companies with direct sales activities, for example, could use the Web as a distribution channel by supporting all phases of EC from contacting to contracting. Furthermore, multimedia presentation offers rich opportunities to describe complex products. Web applications can offer powerful software that helps to tailor products or product bundles (customer decision support). Next, the Web is a global medium, that enables insurers to enter new markets all over the world in a simple but efficient way. And finally, as every information system, Web systems can reduce costs, too, for example by facilitating administrative processes. Web strategy is introduced. Before these potentials will be discussed with respect to the LVM's situation, the current Web usage in the German insurance industry shall be investigated.

\section{State of Web usage in the industry}

In a Web survey conducted during the project at the end of 1997, about 75 Web sites of German insurance companies were identified. The comparison with the numbers of 1996, when 50 companies were present on the Web (Kortzfleisch, Winand 1997), shows, that Web presence in the insurance industry is increasing rapidly. The 75 Web sites identified have been investigated for their information content and features. The results can be summed up like this:

- Most companies use the Web as electronic store front. All companies offer enterprise presentations, a lot of companies offer product information in some way. But only few companies give in depth information on products, including examples of risks, legal obligations and so on. In most cases, the Web sites appear to be nothing else but an "electronic pamphlet".

- Only a few companies make use of the interactive features of the Web. Most companies offer simple email services, of course. But response to e-mails is typically paper based. This media break underscores that the Web is not fully integrated into information and organisational infrastructure. Direct transaction processing and dynamic generation of information are exceptions.

- Less than three companies allow electronic contracting. "Less than three" means that even though electronic contracting was offered, the Web form was just a trigger to the traditional process. The contracting itself was paper based in part or at least accompanied by paperwork.

Another striking result of the Web survey was no perceivable distinction of Web sites according to the business strategies of the companies. For example, the great players in the market would have been expected to use the Web as an interface to international markets. Consequently, Web sites should have been multilingual at least. Or insurers with major direct sales activities would have been expected to use the Web as a medium for market transactions. Web sites should then offer extensive customer information, decision support and electronic contracting. But none of these expectations was confirmed convincingly by the Web survey. The findings can be summed up by the hypothesis that most German insurance companies had not defined or at least implemented a Web strategy clearly supporting their business strategy at that time. 


\section{Web strategy development}

The success of a Web site is not primarily determined by technical or layout qualities. Because of the high visibility and comparability of a Web site, technical and layout qualities can be adapted very soon by competitors. Thus, a sustainable competitive advantage can hardly be achieved through those qualities. In contrast, a decisive success factor of a Web presence is seen in the link between business and marketing strategy on the one hand and Web strategy on the other (Lederer et al. 1997). A Web strategy embedded in and aligned with an overall marketing strategy can hardly be imitated on the short run.

\section{The link between marketing strategy and Web strategy}

LVM is a medium seized player in the German insurance market. The company is well established in numerous regions and covers various market segments. The company has a good reputation, especially in car insurance. LVM is the third supplier on the German market in terms of volume. Nevertheless, the enterprise does not have the size and capacity to compete successfully on foreign markets.

Because of the fierce competition in the national market, LVM intends to bind their customers closer to the company through a customer focused strategy. LVM is neither willing to compete solely on price (cost leadership strategy) nor on very specific market segments (niche strategy). The customer focused strategy instead combines attractive prices, a full set of services all around the customer's needs and intensive customer care in a broader set of market segments.

The customer focused strategy carried out perpetuates a central strengths of LVM. The LVM has at its disposal a large network of (legally) autonomous sales agents distributed over most parts of Germany. Those agents are called "Vertrauensleute", trusted agents, indicating that there is a strong personal relationship between agent and customer. Through this network of agents customers are addressed and looked after personally. Agents are able to give help immediately without much bureaucracy if needed.

To expand customer orientation to a full customer focused strategy, requires customer specific products or product bundles that can be tailored to the customers' needs, increased personal customer care, empowering agents, keep costs and prices low. Direct implications of the customer focused strategy on the Web strategy are:

- The Web site must increase service and provide a clear value for the customer,

- it should enhance customer care,

- it should make use of the new sales channel "Web" without excluding the agents,

- this means that the Web site must not offer electronic contracting in order not to corrupt the existing sales structure,

- instead it should empower the agents.

These implications guided the process of planning the objectives, purpose and scope of the Web site.

\section{Objectives of the Web site}

After having identified a list of potential benefits of the Web usage, the following objectives for the LVM Web site were defined:

- improve reputation through technological leadership,

- improve advertising,

- reduce response and delivery times,

- enhance services, esp. access in place and time, response speed, value added services,

- intensify customer relationships and loyalty,

- facilitate co-operation with sales agencies,

- improve individuality,

- reduce transaction costs by removing or simplifying routine administration work,

- give access to (new) markets.

Some of the objectives are very general like those aiming at image, advertising and transaction costs. Advertising was identified as the most important objective. The Web should offer a comprehensive presentation of the complex product "insurance" through it's multimedia qualities and "infotainment" to attract customers.

Other objectives, such as those concerning response and delivery times, service, customer loyalty, individuality and co-operation with sales agents were directly derived from the customer focused strategy. These objectives are not independent, since the provision of individualised information and of services with a clear value proposition (Kambil et al. 1996) bind the customers to the enterprise and thus improve customer loyalty. 
There is still another objective that has to be explained: "Access to markets" denotes the idea that the Web enables sales agents to improve their care for a regionally dispersed group customers. This is especially important in regions with only a few sales agents, so that (remote) customers can only seldom be looked after personally.

\section{Scope of the Web site}

The following list of information contents and functions defines the scope of the Web site:

- Introduce the enterprise,

- present products with detailed information on all kinds of risks/insurance policies,

- entertain,

- distribute individualised customer information,

- give customer access to his insurance data,

- respond to online customer requests,

- deliver forms, offers, and policies,

- accept claims statements,

- support direct communication between sales agent and customer,

- exchange information between sales agent and head office.

One important underlying idea was to improve services by providing customer specific information. Since information resources are collected and stored at the head office, and the knowledge about customers, their situation, and their needs is located at the agencies, intense co-operation is required. This co-operation should be facilitated by the Web application again.

\section{Interfaces to organisation and information infrastructure}

Integration issues have not been investigated so far, since the proposed Web site is still available only as a prototype. But the main challenge of integration is obvious: The Web site has to be integrated with databases and processes at the head office as well as with the sales agent's infrastructure.

\section{Web application design}

During Web application design the parameters content, features, layout, and technology have to be defined aligned with the Web strategy. Table 2 gives a summary of possible implementations of the conceptual design parameters (also see Kreileder, ABmann 1997).

\begin{tabular}{|c|l|}
\hline Design parameter & \multicolumn{1}{c|}{ Examples } \\
\hline Content & - Enterprise information, \\
& - Product descriptions and price, \\
& - Background information, \\
& - Policy data, \\
\hline Graphical design & - Gaims examples. \\
\hline Features & - Usaphical presentation, \\
& - Animaterface (orientation, navigation, use), \\
\hline & - Customer inquiry / problems management, \\
& - Pamphlet and offer delivery, \\
& - Eroduct calculation and configuration, \\
& - Claims declaration, \\
& - Changes in personal and policy data, \\
& - Individualised counselling (customising, filtering), \\
& - Entertainment. \\
\hline
\end{tabular}

Table 2: Design parameters of Web sites 
The LVM Web application incorporates all of the examples above to a certain extent. Most important are those parameter choices, that directly support the customer focused strategy.

\section{Content}

The LVM application offers static and dynamic information. Information on the enterprise and it's products is only static. The user can browse the information according to his preferences. Choices to pre-select a view to the information are not offered. Other information is customised to the user. It is generated dynamically out of a data base according to the user profile. Such information are the customer's personal data, contracts and policies. Other individualised information is actively (push) distributed to the customers, for example, if the customer has an insurance policy for a car manufactured by BMW, information about this car, technical problems, test reports, etc. Dynamic information is only offered to registered users, i.e. the owners of policies.

\section{Features}

The LVM application offers a lot of standard facilities such as delivery of pamphlets and, the calculation of products, counselling, and entertainment. Counselling and entertainment is close related in the so called "Sorglos-Test" ("care-free-test"), which considers the customer's personal risk situation and a corresponding bundle of policies optimised in price and service. Another entertainment features to attract new customers is a quiz. The questions asked by the system refer to the information on the Web site, so that users taking part in the quiz have to investigate the site in detail.

Probably the most important feature is only offered to registered customers. For them, a direct communication channel to their sales agent is established. This channel includes mailing facilities as well as predefined forms that trigger standard business incidents, such as claims declaration and policy changes. The forms comprise all the data necessary to fulfil the requested transaction. The sales agent only has to trigger the procedure, that is executed at the head office. The agent is then continually informed about status and progress of the procedure. He is the interface and the person to turn to for the customer. Only changes of personal data, the customer's address for example, can be made without explicit involvement of the sales agent.

\section{Layout}

With respect to the layout, there is a distinction between features and content that are public, and those customised to specific users/customers that are pre-registered. The public parts of the application are designed according to more general layout guidelines for a professional and innovative presentation. There is no strict corporate design but a link to it, as the basic enterprise colours and the central logo are used.

More interesting than the public areas of the application are the individualised content and features offered to the registered customers. The entire information contents and all features are organised around a central metaphor, the "virtual desktop". The desktop metaphor implies, that the customer is offered a full working environment supporting him in arranging his insurance affairs. The function of this metaphor is on the one hand to improve orientation and on the other hand to facilitate navigation and access to the application features. Figure 3 shows a sketch of the graphical representation of the "virtual desktop".

When the customers opens his desktop, a photo of his sales agent is displayed in the sales agent-area. He can access all information about his agent by a mouse-click onto this photo. When clicking onto the mail buttons a list of incoming or outgoing mail, respectively, is displayed like in a typical mail system. The folders at the left bottom of the desktop are generated according to the customers policies. Each folder contains all information about a current policy. If a policy is opened, the work aids area offers tools and forms to treat a policy. For example, a claims declaration form can be used and sent to the sale agent (logically) and the head office (physically), respectively.

The pinboard is a kind of individualised bulletin board, that contains information of general relevance to the customer. All bits of information are represented like notes pinned to a wall. They are displayed in the display area on a mouse-click. Figure 5 shows the "virtual desktop" layout used for the prototype. Displayed is the pinboard with (links to) bit of information for a customer assumed to be the owner of a BMW car. 


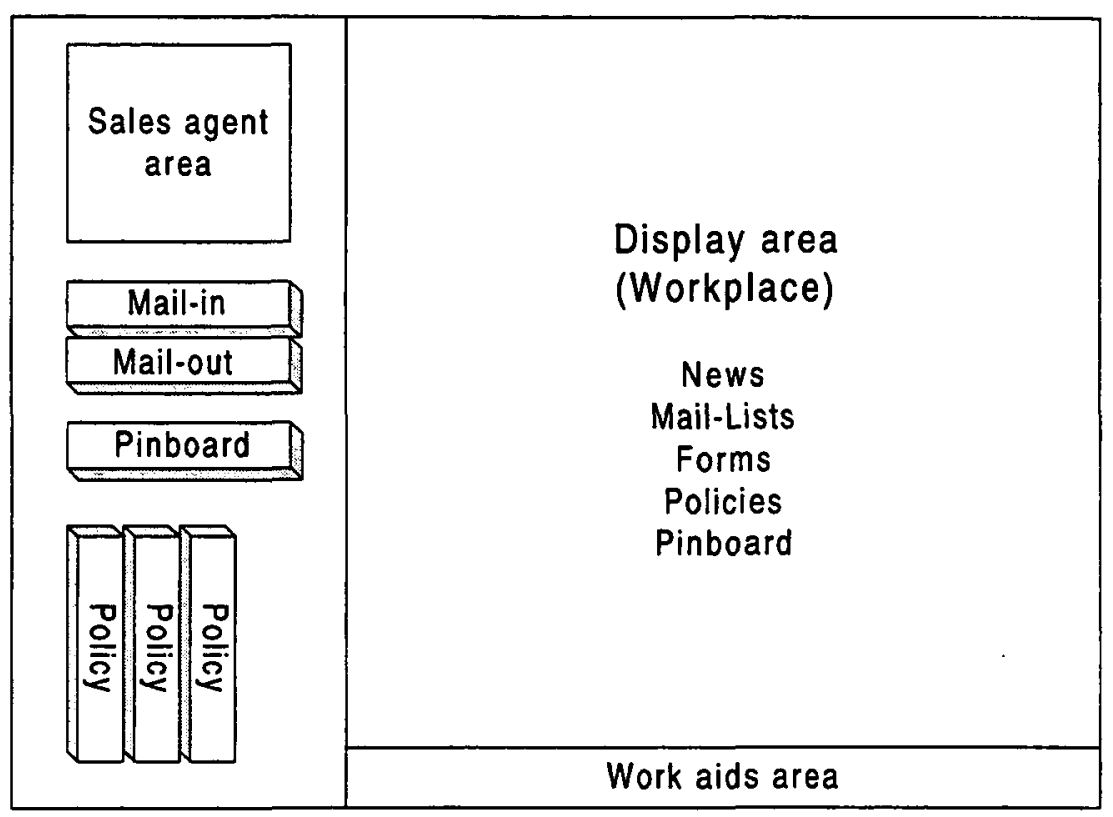

Figure 3: Concept of the virtual desktop

\section{Technology}

The technology for the application has not yet been decided, since LVM is just changing their technical infrastructure. The Web application shall be integrated into the new LVM agency system, that will be based upon an object-oriented architecture. But as the new infrastructure is still in development, the technical parameters of the Web application have not been decided definitely.

The prototype that has been built to prove the concept is based on a Microsoft platform. The Microsoft Information Server has been used as a Web server and Microsoft Access as the database management system. For prototyping purposes these technologies have proved to be valuable. The Active Server Pages offered by the Microsoft Web server and easy integration of Web and database server, facilitated development. Nevertheless, this technological configuration does not have strong implications for the running system. For example, Microsoft Access does not appear to be the right data base management system to handle the transaction volumes for only a part of the 6.5 billion policies of the 2.8 million customer.

Figure 2 describes the development cycle of Web applications. The preceding description of the planning and design phase refers to the joint development of a prototype which is not yet fully implemented. The prototype actually represents a redesign of the initial Web site. The following description of the integration and evaluation phases refer therefore to the initial design.

\section{Integration}

Development and maintenance of the initial Web presence had been outsourced to a service provider. The Web application ran fully independent of LVM's IT infrastructure. While this was advantageous from a security point of view, it not only restricted the functionality of the Web application to a static information site, it also made any change of the content a cumbersome process. Change requests had to be send to the service provider and checked afterwards. The organisational and technical separation between service provider and LVM was quickly perceived as a major disadvantage and totally inadequate for a dynamic Web presence.

The redesigned application will be controlled and maintained internally, although parts of the development may again be outsourced. A linkage to the operational database management systems is envisioned but not yet realised.

\section{Evaluation and monitoring}

The critical assessment of the initial Web presence and the mentioned analysis of the state of the Web usage in the industry, both give evidence to evaluation and monitoring activities which were the basis for the redesign. 


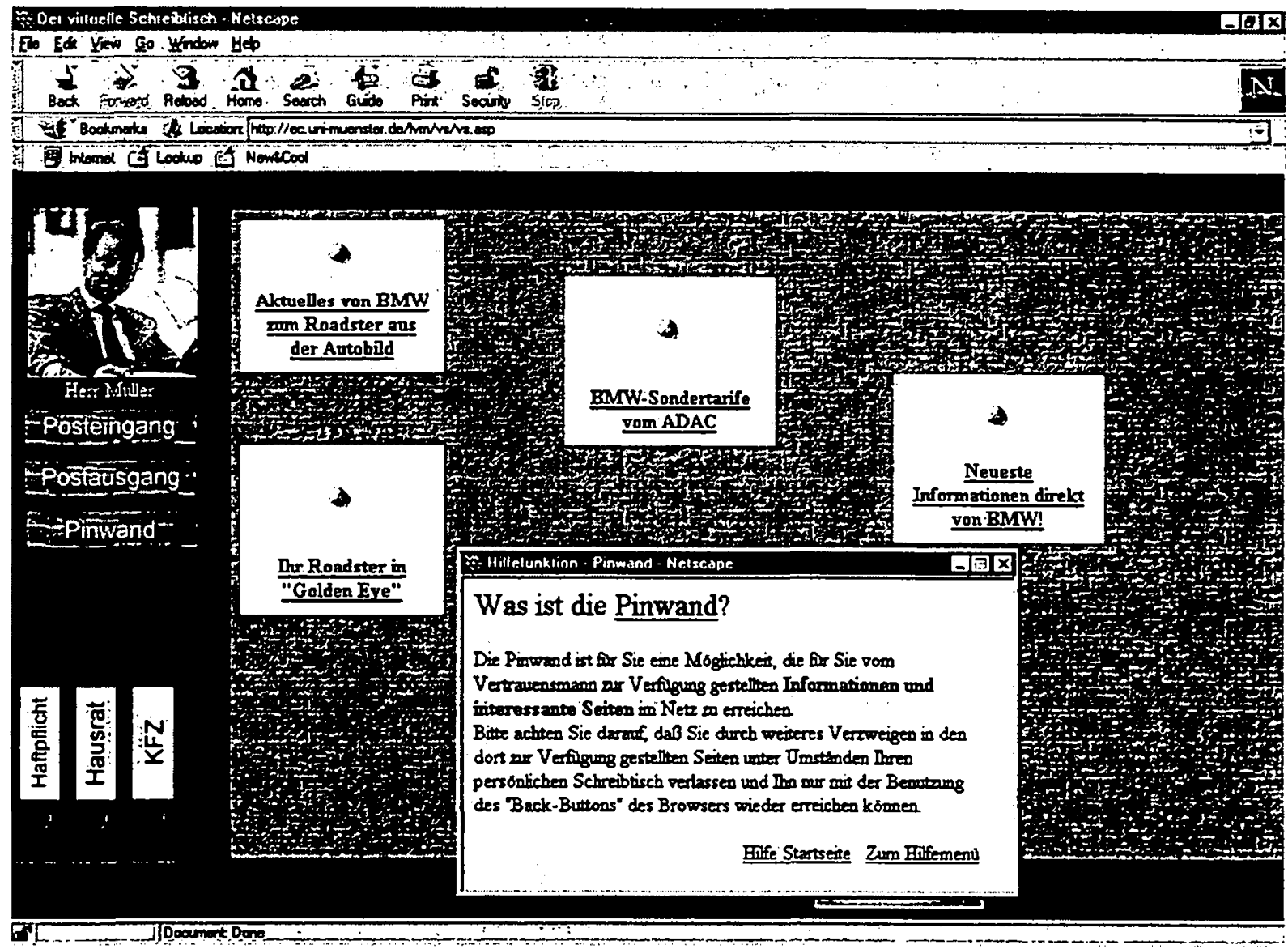

Figure 4: Layout of the virtual desktop

\section{CONCLUSIONS}

The analysis of the LVM case underscores the assumption that a successful Web presence requires much more than a me-too strategy or a pure informational Web site. The example shows how properties of the Web as a new medium and the strategy can be aligned in order to develop specific value propositions for respective target groups. These value propositions clearly go beyond the conventional wisdom of product fit for the Web: the functional areas that have been selected are neither easy to describe products nor direct sales activities. They reflect, however, the company's strategy and build on functional properties of the technology. If these value propositions are related to the core competencies, it might even be possible to achieve sustainable competitive advantages in a medium which facilitates imitation more than any medium before.

However careful and innovative a solution might be, given the dynamics and volatility of the medium Web, a continuous process of monitoring and learning has to be initiated in order to gain and/ or keep a competitive edge.

\section{REFERENCES}

Kassaye, W. (1997) "Global Advertising and The World Wide Web", Business Horizons, Vol. 40, No. 3, pp 33-42.

Klein, S. (1998) "Konzepte und Vorgehensmodelle für die Web-Evaluation", in: Korte, Werner B.; Reinhard, Ulrike (eds.): who is who in electronic commerce, whois verlags- und vertriebsgesellschaft, Heidelberg, pp 200-209.

Kambil, A.; Ginsberg, A. \& Bloch, M. (1996) "Reinventing Value Propositions", Working Paper IS-96-21, New York University, New York.

Kortzfeisch, H. F. O. \& Winand, U. (1997) "Tele-Insuring: der Beitrag neuer Kommunikations- und Infromationstechniken (KIT) zur Verbesserung der Kundenorientierung im Versicherungsbereich", Die Betriebswirtschaft, Vol. 57, No. 3, pp 338-356. 
Kreileder, M.; Aßmann, K. (1997) "Insurance online: Die meisten kamen aus Neugier". In: Versicherungswirtschaft, Vol. 16.

Lederer, A. L.; Mirchandani, D. A. \& Sims, K. (1997) "The Link between Information Strategy and Electronic Commerce", Journal of Organisational Computing and Electronic Commerce, Vol. 7, No. 1, pp 17-34.

Schoder, D.; Strauß, R. E. \& Welchering, P. (1998) "Electronic Commerce Enquête 97/98 - Executive Research

Report: Empirische Untersuchung zum betriebswirtschaftlichen Nutzen von Electronic Commerce für Unternehmen im deutschsprachigen Raum". 